\title{
Pulse methylprednisolone therapy for arthritis causing muscle weakness
}

\author{
E Michael Shanahan, Malcolm D Smith, Michael J Ahern
}

The occurrence of multiple autoimmmune conditions in the same patient is a well recognised phenomenon. In this case, we present a patient with an inflammatory arthritis (late onset rheumatoid arthritis) with previously unrecognised myasthenia gravis that was unmasked by high dose intravenous pulse methylprednisolone succinate used to treat the arthritis.

\section{Case history}

A 78 year old man was admitted to hospital complaining of progressive small joint pain, stiffness and swelling. This had been present for four weeks and was on a background of several months of systemic symptoms including weight loss of approximately $14 \mathrm{~kg}$, fatigue, anorexia, intermittent dysphagia, and headaches. In addition he had suffered from intermittent mid-thoracic spine, shoulder and left hind foot pain and stiffness.

Two months previously he had been investigated for these constitutional symptoms with plain radiographs, a bone scan, abdominal ultrasound, erythrocyte sedimentation rate (ESR), $C$ reactive protein (CRP), serum electrophoresis, urinary Bence Jones protein, uric acid, prostatic specific antigen (PSA), rheumatoid factor, iron studies, liver function tests, complete blood picture, and serum calcium. All these investigations were normal except for a PSA of $50 \mu \mathrm{g} / 1$ (normal $<4$ ), radiographs showing mild osteoarthritis of the thoracic and lumbar spine, a CRP 80 $\mathrm{mg} / \mathrm{l}$ (normal<6) and an ESR of $47 \mathrm{~mm} 1 \mathrm{st} \mathrm{h}$. The increased PSA had been investigated further with digital rectal examination, prostatic ultrasound and biopsy, which showed moderate prostatic hypertrophy, with no evidence of malignancy. A diagnosis of polymyalgia rheumatica was considered, and a two week trial of prednisolone $20 \mathrm{mg} /$ day instituted with no benefit, leading to a weaning (over two further weeks) of the corticosteroid treatment.

His past medical history consisted of controlled hypertension and asthma, treated with prazosin $5 \mathrm{mg}$ twice daily, verapamil $180 \mathrm{mg}$ daily, inhaled salbutamol and beclomethasone twice daily.

Physical examination revealed an acute symmetrical polyarthritis with active synovitis of the small joints and tendon sheaths of his hands and feet with marked peripheral pitting oedema of his hands. In addition his wrists, shoulders and ankles were tender. The remainder of the examination including muscle power was normal.

Investigations revealed; ESR $54 \mathrm{~mm} 1 \mathrm{st} \mathrm{h}$, haemoglobin $104 \mathrm{~g} / \mathrm{l}$, PSA $29 \mu \mathrm{g} / \mathrm{l}$, CRP 42 $\mathrm{mg} / \mathrm{l}$, and rheumatoid factor $27 \mathrm{IU} / \mathrm{ml}$ (normal $<40$ ), with a negative ANA. Radiographs of the hands and feet showed minor degenerative changes only. The diagnosis of an acute inflammatory arthropathy was made on clinical grounds, with a provisional diagnosis being made of late onset rheumatoid arthritis (RA). Polymyalgia rheumatica and remitting, seronegative, symmetrical, synovitis with pitting oedema $\left(\mathrm{RS}_{3} \mathrm{PE}\right)$ syndrome were considered in the differential diagnosis.

In view of the poor response to low dose oral corticosteroids, treatment was started with high dose intravenous methyl-prednisolone succinate $(1000 \mathrm{mg}$ in $100 \mathrm{ml}$ normal saline over 20 minutes) followed by methotrexate 7.5 mg once a week and oral prednisolone $15 \mathrm{mg}$ daily. The day after the initial pulse there was considerable improvement with less pain and a reduction in swelling but the patient was still disabled by continuing symptoms.

After a second intravenous dose of methylprednisolone succinate his arthritis improved markedly but he became acutely dysarthric and dysphagic for both fluids and solids. Bilateral ptosis, weakness of the neck flexor muscles and orbicularis oris and oculi were noted. The diagnosis of myasthenia gravis was considered and a tensilon test performed. This was unequivocally positive with a rapid improvement in symptoms within seconds of the administration of edrophonium.

Regular pyridostigmine was started and he rapidly recovered. Azathioprine $50 \mathrm{mg}$ twice daily was substituted for methotrexate in view of its reported efficacy in the treatment of myasthenia gravis, ${ }^{1}$ and the reminder of his admission was uneventful. Computed tomography showed no evidence of a thymoma or bronchogenic neoplasm. He was discharged with a complete resolution of his myasthenic symptoms with considerable improvement in his joint symptoms.

Subsequent antibody testing revealed antiacetylcholine receptor antibodies of $4910^{10}$ mol bungarotoxin bound/1 (normal <3).

Follow up three months after discharge revealed ongoing tenderness and some swelling 
of the wrists but overall considerable improvement in joint pain signs and symptoms, and no recurrence of myasthenic symptoms. His CRP had fallen to $4 \mathrm{mg} / \mathrm{l}$.

\section{Discussion}

As an autoimmmune condition myasthenia gravis is known to be associated with a number of inflammatory arthropathies including $\mathrm{RA}^{2}$ and systemic lupus erythematosus (SLE). ${ }^{3}$ Although the series are small, there is evidence to suggest that approximately $4-7 \%$ of people with myasthenia gravis have RA and up to $8 \%$ have SLE. There are also case reports of the coexistence of myasthenia in patients with scleroderma, ${ }^{4}$ ankylosing spondylitis, ${ }^{3}$ psoriatic arthritis $^{5}$ and juvenile chronic arthritis. ${ }^{6}$ In addition, arthralgias have been noted during the active phase of myasthenia gravis. ${ }^{2}$ Although the precise diagnosis of our patient's arthritis remains unclear, the distribution, mode and age of onset suggest a diagnosis of late onset RA. The fact that the arthritis had not responded to the earlier dose of corticosteroids is not surprising as the peripheral arthritis was not prominent in the early stages and also because up to $40 \%$ of RA patients may not respond to low dose oral corticosteroid therapy. ${ }^{7}$

Prednisolone is a well recognised treatment for both RA and myasthenia gravis. In myasthenia gravis, corticosteroids are known to reduce the titre of acetylcholine receptor antibody. ${ }^{8}$ They may also improve neuromuscular transmission, have a role in the production of acetylcholine receptor and help stabilise postsynapatic membranes. ${ }^{9}$ However, the association between an aggravation of myasthenic symptoms and the introduction of corticosteroids is well reported, occurring in up to $50 \%$ of patients, particularly at higher doses. ${ }^{10}$ In the acute situation exacerbations usually occur from day one to three weeks into treatment. The cause may be related to interference with excitation-contraction coupling and blockage of open ion channels in the acetylcholine receptor, ${ }^{11}{ }^{12}$ but the precise mechanisms are unclear. In the chronic situation corticosteroid induced myopathy can aggravate pre-existing myasthenia gravis. To our knowledge there are no previous reported cases of pulse methylprednisolone as treatment for arthritis revealing myasthenia gravis.

\section{The lessons}

- There is a potential association of myasthenia gravis and rheumatoid arthritis.

- The presence of muscle weakness should suggest the possibility of myasthenia gravis.

- Corticosteroids, which may be used in the treatment of both rheumatoid arthritis and myasthenia gravis, can cause an acute onset or aggravation of the symptoms of myasthenia gravis.

1 Palace J, Newson-Davis J, Lecky B. A randomised double-blind trial of prednisolone alone or with azathioprine in myasthenia gravis. Neurology 1998;50:1778-83.

2 Thorlacius S, Aarli JA, Riise T, Matre R, Johnsen HJ. Associated disorders in myasthenia gravis: autoimmune diseases and their relation to thymectomy. Acta Neurol Scand and their relation

3 Vaiopoulos G, Sfikakis PP, Kapsimali V, Boki K, Panayiotidis P, Aessopos A, et al. The association of systemic lupus erythematosis and myasthenia gravis. Postgrad Med J 1994;70:741-5.

4 Bhalla R, Swedler WI, Lazzarevic MB, Ajmani HS, Skosey JL. Myasthenia gravis and scleroderma. J Rheumatol 1993 20:1409-10

5 Brand CA, Littlejohn GO. Psoriatic arthritis and myasthenia gravis. J Rheumatol 1984;11:244.

6 Bhetty EM, Kalla AA. Coexistence of myasthenia gravis and seropositive juvenile chronic arthritis. Clin Exp Rheumatol 1992;10:499-501.

7 Van Gestel AM, Lann RFJM, Haagsma CJ Van De Putte LBA, Van Riel PLCM. Oral steroids as bridge therapy in RA patients starting with parental gold: a randomised RA patients starting with parental gold: a randomised 1995;34:347-51.

8 Tindall RSA. Humoral immunity in myasthenia gravis: effects of steroids and thymectomy. Neurology 1980;30: $554-7$

9 Pourmand R. Myasthenia gravis. Dis Mon 1997;43:65-109

10 Barrons RW. Drug-induced neuromuscular blockade and myasthenia gravis. Pharmacotherapy 1997;17:1220-32.

11 Wittbrodt ET. Drugs and myasthenia. Arch Intern Med 1997; 157:399-408.

12 Miller RG, Milner-Brown HS, Mirka A. Prednisoloneinduced worsening of neuromuscular function in myasthenia gravis. Neurology 1986;36:729-32. 